\title{
Sphingolipids, Lipid Rafts, and Giardial Encystation: The Show Must Go On
}

\author{
Tavis L. Mendez ${ }^{1,2}$ - Atasi De Chatterjee ${ }^{1,2}$ - Trevor Duarte ${ }^{1,2}$ Joaquin De Leon ${ }^{1,2}$. \\ Leobarda Robles-Martinez ${ }^{1,2} \cdot$ Siddhartha Das ${ }^{1,2}$
}

Published online: 11 July 2015

(C) Springer International Publishing AG 2015

\begin{abstract}
Sphingolipids are sphingosine-based phospholipids, which are present in the plasma and endomembranes of many eukaryotic cells. These lipids are involved in various cellular functions, including cell growth, differentiation, and apoptosis. In addition, sphingolipid and cholesterol-enriched membrane microdomains (also called "lipid rafts") contain a set of proteins and lipids, which take part in the signaling process in response to intra- or extracellular stimuli. Recent findings suggest that sphingolipids, especially glucosylceramide, play a critical role in inducing encystation and maintaining the cyst viability in Giardia. Similarly, the assembly/disassembly of lipid rafts modulates the encystation and cyst production of this ubiquitous enteric parasite. In this review article, we discuss the overall progress in the field and examine whether sphingolipids and lipid rafts can be used as novel targets for designing therapies to control infection by Giardia, which is rampant in developing countries, where children are especially vulnerable.
\end{abstract}

Keywords Giardia · Lipid · Sphingolipid · Lipid rafts · Encystation · Cyst

This article is part of the Topical Collection on Protozoa (Giardia)

Siddhartha Das

sdas@utep.edu

1 Infectious Disease and Immunology Cluster, The Border Biomedical Research Center, University of Texas at El Paso,

El Paso, TX 79968, USA

2 Department of Biological Sciences, University of Texas at El Paso, 500 W. University Avenue, El Paso, TX 79968-0519, USA

\author{
Abbreviations \\ CTXB cholera toxin B subunit \\ CWP cyst wall protein \\ ESV encystation-specific vesicle \\ gGlcT1 giardial glucosylceramide transferase-1 \\ gLR giardial lipid raft \\ GM1 mono-sialo ganglioside \\ LR lipid raft \\ SL sphingolipid
}

\section{Introduction}

The enteric protozoan Giardia lamblia (also known as Giardia intestinalis and Giardia duodenalis) is responsible for waterborne diarrhea or giardiasis, worldwide [1]. In the USA, giardiasis is particularly prevalent in day care centers and among hikers and campers. According to a survey conducted by the Centers for Disease Control and Prevention (CDC), approximately 15,000-16,000 cases of giardiasis were reported in the USA between 2011 and 2012, with the majority recorded in the northwest states. Furthermore, it was observed that children between the ages of 1 and 4 years are most vulnerable to the infection [2]. Earlier CDC survey data (for 20062010) indicated about 19,000 cases of giardiasis in the USA, with northern states affected most [3, 4]. Giardiasis is also not uncommon in Western European countries. For instance, Ekdahl and Andersson [5] reported that giardiasis in Sweden is associated with international travel, immigration, and adoption. People who travel to the Indian subcontinent and to East and West Africa are more susceptible to giardiasis. For immigrants in Sweden, the incidence of giardiasis is high among people from Afghanistan and Iraq. 
Giardia has a simple biphasic life cycle-i.e., replicative trophozoites and relatively dormant cysts. While cysts are responsible for the transmission of Giardia through sewage-contaminated water, trophozoites colonize the upper portion of the human small intestine, which can cause symptomatic or non-symptomatic giardiasis. The hardy, water-resistant cysts undergo "excystation" in the stomach, and newly emerged trophozoites adhere to the surface of the small intestine and initiate the disease process. When conditions change, trophozoites become cysts (called "encystation") and move downstream into the large intestine. The cysts are then eliminated from the body in the stool to infect a new host [6]. Development of methodologies for in vitro encystation and excystation [7-10] has revolutionized the research on Giardia over the past 40 years. The ability to culture and differentiate this parasite in the laboratory has allowed investigators to study the gene/ protein expression, metabolic pathways, and biology of this fascinating unicellular organism. Giardia trophozoites are flagellated, binucleate, and 12-15 $\mu \mathrm{m}$ long (Fig. 1a). They are non-invasive, vibrant, and replicative, and contain a ventral disc made of cytoskeleton proteins, which not only provides support but also allows the trophozoites to attach to intestinal cell walls, which is critical for initiating the infection in the gut [11-14]. The cysts (Fig. 1d), on the other hand, are mostly oval shaped, relatively dormant (7-10 $\mu \mathrm{m}$ long), and composed of thick, osmotically resistant cyst walls (0.3$0.5 \mathrm{~mm}$ thick). Giardial cysts have tetranucleatecontaining basal bodies and axonemes, which are close in proximity to nuclei and fragmented discs [15]. The cyst walls are made of proteins and $\beta-(1,3)-\mathrm{N}-$ acetylgalactosamine homopolymer, which provides a solid support to the cyst wall [16-19].

\section{Encystation by Giardia: Stories Told by Genes and Proteins}

As mentioned above, the ability to induce encystation in culture (i.e., in vitro encystation) has been instrumental in elucidation of the mechanism of giardial encystation. Transcriptome analyses during encystation of this parasite have revealed upregulation of the genes for cyst wall proteins (CWPs), protein kinases, metabolic pathways, and unidentified hypothetical proteins [20]. Likewise, the Serial Analysis of Gene Expression (SAGE) study has indicated that about 71 transcripts have been upregulated specifically during excystation and 42 transcripts at the time of encystation. These transcripts include the mRNAs for CWPs, metabolic and signaling molecules, kinases, phosphatases, proteases, and cell surface proteins [21]. The results of proteomic analyses [22] are similar to the transcriptomic data $[20,21]$ and reveal that proteins involved in cytoskeleton function, NIMArelated kinases (NEKs), CWPs, glycolysis/metabolic enzymes, and proteins linked to protein folding are upregulated during encystation. Variable surface proteins (VSPs) that are predominantly expressed in trophozoites decrease with encystation, and the proteome profile of late-encysting cells exhibits protein compositions similar to those of non-encysting trophozoites [22].

Since cyst formation is an important step in the giardial life cycle, many investigators have devoted considerable amounts of time and effort to study this process at the cellular and molecular levels [23]. Three encystation-specific cyst wall proteins (CWP-1, -2, and -3) are expressed and concentrated within encystationspecific vesicles (ESVs) before they are transported to the cyst wall [15]. CWP-1, -2 , and -3 are acidic proteins (molecular mass $\sim 26, \sim 39$, and $\sim 27 \mathrm{kDa}$, respectively) containing similar domains, including N-terminal signal sequences and five tandem leucine-rich repeats [24, 25]. Recent studies have suggested that all three CWPs are essential for forming ESVs, and that CWP-2 functions as an aggregation factor to regulate ESV formation by interacting with CWP-1 and CWP-3 via conserved regions [26]. Overexpression of $g M y b 2$ results in an increase of CWP1 expression and cyst formation by Giardia [27]. UPF1, a conserved non-sense-mediated mRNA decay factor, has also been shown to regulate cyst production by decreasing $c w p$ and gmyb2 transcripts [28]. In addition to CWPs, Myb2, and UPF1, several other genes and proteins have been identified; however, their precise roles in encystation and the mechanisms by which they interact with various encystation components are not known. Several encystation-specific signaling molecules-e.g., cAMP-dependent protein kinase (PKA), protein phosphatase (PP2A), ERK/MAPK, and protein kinase $\mathrm{B} / \mathrm{Akt}$ - have been identified and shown to be involved in inducing encystation [23]. At the time of excystation, intracellular proteases, calcium, and other signaling proteins are involved in digesting the cyst wall to facilitate the emergence of two new metabolically active trophozoites [29]. It has been proposed that the cross-talk between PP2A and PKA and calmodulin signaling are important for excystation [23].

Encystation in Giardia is initiated by the biogenesis of the ESVs [30]. The induction of ESV synthesis and its direct link with the process of encystation were first demonstrated by Dr. Frances Gillin and her group from the University of California at San Diego [31]. Since then, about 160 papers have been published on ESV and encystation, and a new area of research has emerged on protein trafficking and secretory pathways in this early-diverging eukaryote [30, 32-34]. Encystation 
Fig. 1 Morphological transformation of trophozoites to cysts (encystation). Upper panel (a-d): differential interference contrast (DIC) images of nonencysting and encysting trophozoites and water-resistant cysts. As encystation progresses, trophozoites become round shaped in the late-encysting phase and finally form cysts. Middle panel (e-h): The biogenesis of encystation-specific vesicles (ESVs) (red) is visible in the early and late encystation stages. The ESVs, which are more numerous in the late stages, are preparing to be fused with the plasma membrane (PM). and form a cyst wall (CW). Bar: $5 \mu \mathrm{m}$. Lower panel $(\mathbf{i}-\mathbf{j})$ : Transmission electron micrographs representing a nascent ESV attached to the endoplasmic reticulum (ER) lumen and mature ESVs transported to the plasma membranes. Bar: $500 \mathrm{~nm}$. FL flagella, $V D$ ventral disc

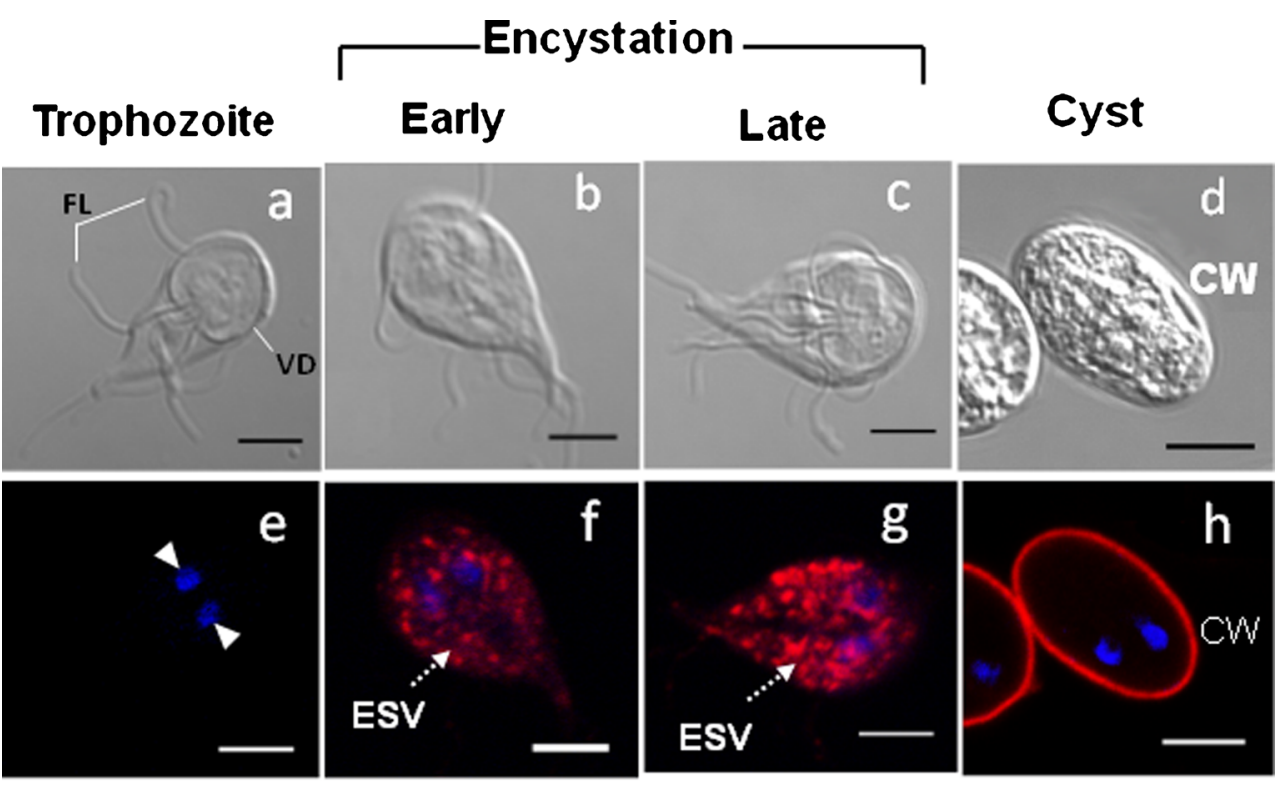

ESV-Attached to ER

ESV-Attached to PM
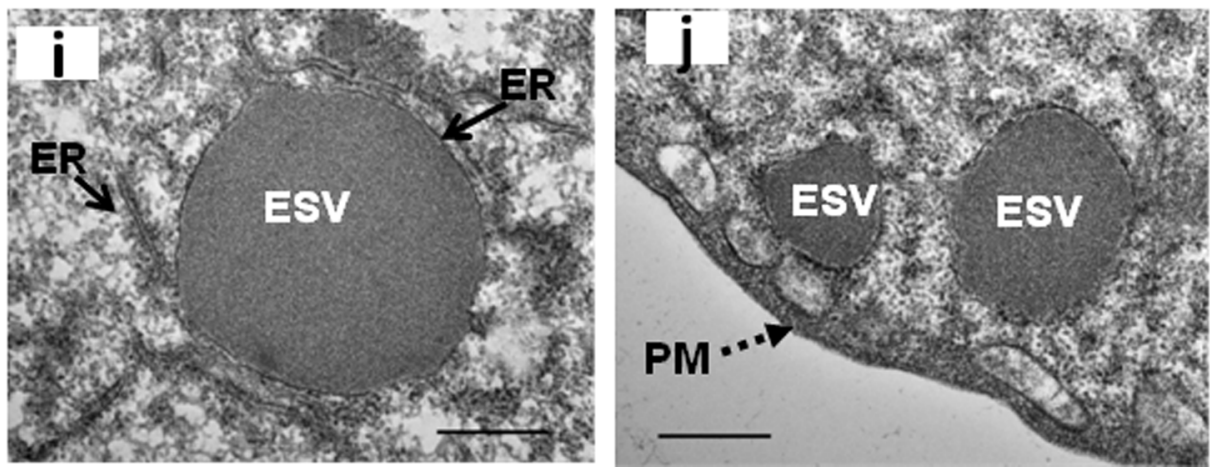

stimuli induce the synthesis of ESVs within 4-6 hours of encystation, which then grow in perimeter and area in the late-encysting phase (i.e., 24 hours) and finally merge with the plasma membranes of trophozoites to lay down the cyst wall (see differential interference contrast [DIC] images in Fig. 1a-d and images immunostained with cyst antibody in Fig. 1e-h). Images from transmission electron microscopy display newly formed ESVs emerging from the endoplasmic reticulum (Fig. 1i) and mature ESVs are preparing to fuse with the plasma membranes (Fig. 1j). ESVs serve as transport vesicles and are involved in carrying CWPs and other cyst wall antigens to the plasma membranes of trophozoites [35, 36]. Bittencourt-Silvestre et al. [37] demonstrated that LY294002 (an inhibitor of PI3 kinase), genistein (which blocks the activity of tyrosine kinase), and staurosporine (which inhibits protein kinase C activity) interfere with ESV biogenesis and cyst production, suggesting that ESV biogenesis is important and a critical step of successful encystation, and interruption of this process by metabolic and pathway inhibitors lowers the production of cysts [37].

\section{Sphingolipids and Encystation}

In 2008, two laboratories independently reported that smallmolecule inhibitors such as D-threo-1-phenyl-2palmitoylamino-3-morpholino-1-propanol (PPMP) and Dthreo-1-phenyl-2-decanoylamino-3-morpholino-1-propanol (PDMP), which are known to inhibit glucosylceramide (GlcCer) synthesis, blocked encystation and cyst production by Giardia $\left[38^{\bullet}, 39 \bullet\right]$. GlcCer synthesis is catalyzed by the glucosylceramide transferase enzyme (GlcT1; also known as glucosylceramide synthase or GCS), which transfers glucose from UDP-glucose to a ceramide acceptor, as shown below.

\section{PPMP or PDMP}

UDP-glucose + ceramide $-\mathbf{X} \rightarrow$ glucosylceramide

Both PPMP and PDMP are widely used to evaluate the roles of GlcCer or other sphingolipids (SLs) in cell division, cytokinesis, and drug-resistance phenomena in cancer cells [40]. However, studies suggest that PDMP can exhibit off- 
target activities. For example, rather than inhibiting only GlcCer synthesis, PDMP can inhibit other enzymes of SL metabolism [41, 42] and calcium influx, as well as membrane functions [43]. PPMP has been shown to block the replication cycle of Giardia trophozoites (i.e., trophozoite duplication) and to generate doublet and triplet cells instead of a singlet trophozoite, suggesting that GlcCer synthesis is linked to cytokinesis [39॰]. Because successful cytokinesis and singlet trophozoite formation are important for trophozoites to enter the encystation cycle [44], researchers have also asked if PPMP could inhibit Giardia encystation. PPMP was found to block encystation by interfering with the expression of CWP1 [40]. Since GlcT1 is an important enzyme of SL metabolism, Hernandez et al. [38 ] analyzed SL genes in Giardia and identified only three putative SL syntheses and two putative metabolic genes. These include two copies of giardial serine-palmitoyltransferase genes (i.e., gspt-1 and gspt-2), one copy of the giardial glucosylceramide transferase- 1 gGlcT1 gene (gglct1), and two sphingomyelinase genes (gsmase $B$ and gsmase $3 b$ ). The transcriptomic analyses indicate that gspt-1 and gspt-2 are transcribed mostly in trophozoites. mRNA expression decreases in pre-encysting and early-encysting cells and increased again in late-encysting cells, as well as in cysts. On the other hand, the expression of gglct1 transcript is low in trophozoites and increases in encysting cells, which correlates with the induction and maturation of ESVs. Interestingly enough, the transcripts of both gsmase $B$ and gsmase $3 b$ increase during encystation.

Although Giardia has the gene for synthesizing GlcCer de novo [38•], it is unable to generate its own ceramide. As the majority of ceramide is taken up from the culture medium or from the small intestinal milieu, some could be generated by breaking down sphingomyelin by Smase enzymes [38•, 45-47]. Exogenously administered fluorescently conjugated ceramide was found to be targeted in the ER/perinuclear regions of trophozoites [48, 49], and in encysting cells it reacted with parallel perinuclear cisternae, which appeared to be a Golgi apparatus $[25,35,36,50]$. This suggests that exogenous ceramide not only serves as a precursor of GlcCer but is also required for organelle biogenesis in Giardia. Various inhibitors and conditions such as ouabain, menadione, $\mathrm{K}^{+}$-depletion, cytochalasin-D, and vinorelbine interfere with ceramide uptake, and it has been proposed that Giardia uses clathrincoated vesicles and actin/microtubule filaments for the uptake and intracellular targeting of ceramide. On the basis of a study by Hernandez et al. [51•], it can be concluded that ceramide, GlcCer, and other SLs are important for ESV biogenesis and cyst production. At the biochemical level, the inhibition of GlcCer synthesis (by PPMP) increased the intracellular ceramide concentration and blocked cell division and cytokinesis [52•]. Inhibition of GlcCer synthesis in Giardia caused structural abnormality, perturbed the localization of clathrin-coated vesicles, and interfered with the synthesis and trafficking of
ESVs. These results support the idea that ceramide endocytosis and GlcCer synthesis are critical for growth, differentiation, and cyst formation.

\section{Glucosylceramide, Sphingolipids, and Cyst Viability}

The ability of Giardia to attach, replicate, and continue its life cycle in the human small intestine is central to the biology and pathophysiology of this parasite. Since Giardia is known not to secrete any toxin or antigen, and no virulence factor has been identified thus far, it is likely that both parasitic and intestinal factors are involved in producing symptomatic and asymptomatic giardiasis. Another important aspect of giardial biology is its ability to switch morphological stages - from trophozoites to cysts - to avoid killing by host defense systems [13, 53-55]. Since GlcCer synthesis is important for trophozoite replication, cytokinesis, and encystation [39•, 52•], and because the generation of viable cysts is absolutely necessary for excystation and establishing infection in the gut [56], Mendez et al. [57•] examined whether GlcT1, which facilitates GlcCer synthesis, regulates ESV biogenesis, encystation, and cyst viability.

At the cellular level, cyst production is mostly dependent on the quality of ESVs, as these vesicles are involved in transporting CWPs to the plasma membranes [55]. Consequently, interrupting the process of ESV biogenesis leads to the generation of incomplete, cryptic, and non-type I cysts $[37,58]$. Therefore, Mendez et al. [57 ] monitored the biogenesis of ESVs in gGlcT1 overexpression, knockdown, and rescue conditions. First, the overall gGlcT1 activity was measured in cell-free extracts, and it was observed that the enzyme activity was upregulated during encystation and increased several-fold in cysts. Although it was noted that gGlcT1 from non-encysting and encysting trophozoites was sensitive to PPMP, the activity in cyst-gGlcT1 was not inhibited by PPMP. This suggests that the characteristics and/or the substrate affinity of gGlcT1 are different in non-encysting trophozoites and cysts. More recently, it was observed that gGlcT1 could utilize both UDP-galactose and UDP-glucose and appeared to function as both GlcT1 and ceramide galactosyltransferase (CGT) enzymes (Robles-Martinez et al., manuscript in preparation). This may offer an explanation as to why gGlcT1 in cysts is not inhibited by PPMP. The modulation of gGlcT1 activity by overexpression and knockdown also affected the ESV structures. Overexpression of gGlcT1 produced enlarged and aggregated ESVs, and knockdown completely blocked the biogenesis of ESVs. Interestingly, when gGlcT1overexpressed cells were transfected with anti-gGlcT1 morpholino oligonucleotide, both the enzyme activity and the ESV synthesis returned to normal. It was noted that gGlcT1 overexpression disturbed the overall cellular lipid homeostasis by increasing the internalization of various lipids, 
Fig. 2 Hypothetical model showing that giardial lipid rafts are activated by various activators present in the small intestine and generate downstream signals that upregulate encystation-specific genes and proteins, as well as giardial glucosylceramide transferase-1 (gGlcT1). gGlcT1, located in the endoplasmic reticulum (ER), uses ceramide (Cer), scavenged by the parasite from its environment, and produces glucosylceramide (GlcCer). GlcCer then induces the biogenesis of encystation-specific vesicles (ESVs). ESVs transport cyst wall proteins to the plasma membranes (PMs) and form the cyst wall (shown in red)

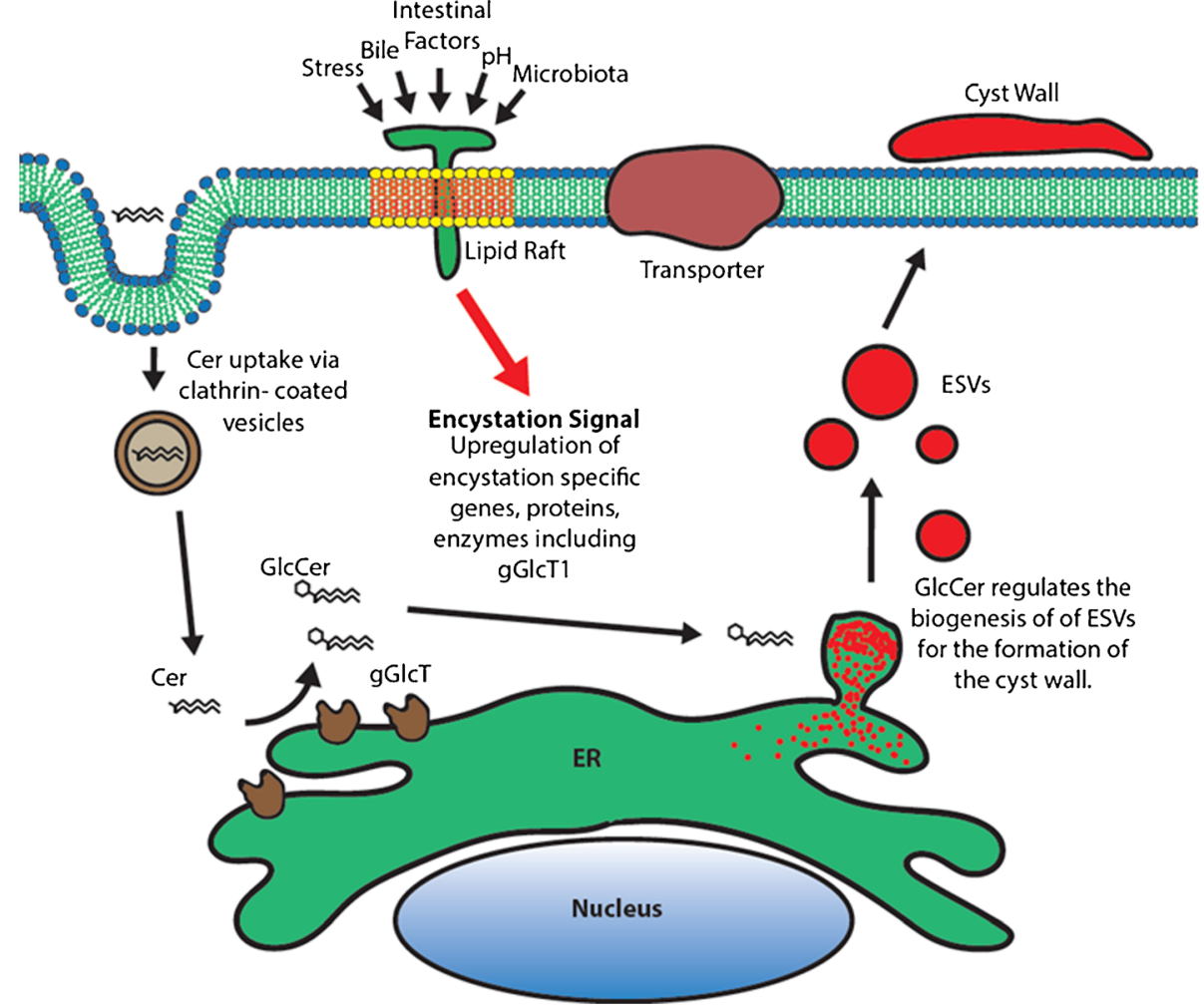

PM fatty acids, and cholesterol. However, the changes of the intracellular lipid balance did not alter the membrane fluidity. Like ESVs, the modulation of gGlcT1 activity also affected cyst structures and viability, as both overexpression and knockdown altered the cyst quality and reduced viability. The rescue treatment, however, produced cysts with wellformed cyst walls [57•]. These studies further support the idea that regulated expression of gGlcT1 is important for the biogenesis of functional ESVs and maintaining cyst viability.

\section{Glycosphingolipid, Lipid Rafts, and Encystation}

Lipid rafts (LRs) are small nanoscale assemblies located in the plasma membrane of an eukaryotic cell and function as "molecular switches" to modulate various cellular functions [59]. These raft microdomains are dynamic in nature and can undergo self-assembly and disassembly during receptorligand interactions and/or raft (caveolae)-mediated endocytosis [60-62].

One of the pertinent questions in giardial biology is how this parasite undergoes encystation in the small intestine and, most importantly, how this parasite senses changes in the environment and responds suitably by transforming into cysts to avoid killing by the factors present in the small intestinal milieu. To answer this question at least in part, it was hypothesized that Giardia, like many eukaryotes, assembles lipid rafts (gLRs), which act as "molecular sensors" to receive and relay extracellular signals downstream of the plasma membrane. Since the majority of eukaryotic LRs are composed of mono-sialo ganglioside (GM1) [63], fluorescently conjugated cholera toxin B (CTXB) was used to identify gLRs in Giardia because CTXB binds specifically to the GM1 glycolipid of LRs. CTXB reactions were found to be localized in the plasma membranes, ventral disc, and caudal flagella of trophozoites. gLRs were also immunostained with GM1 antibody similar to CTXB and showed a similar staining pattern to CTXB. Nystatin and filipin-III, two well-known LR-disrupting agents, and oseltamivir (a viral neuraminidase inhibitor) lowered the CTXB and GM1-antibody staining significantly, indicating that like all other eukaryotic rafts, gLRs also contain cholesterol, and removal of cholesterol destabilized the microdomains [64•].

To establish a possible link between the assembly of gLRs and cyst formation, these drugs were tested on giardial encystation in vitro. The results indicated that the two cholesterol-binding agents blocked ESV biogenesis, as well as the transition of trophozoites into cysts, and generated incomplete and morphologically altered cysts, which could be stained with trophozoite antibody rather than cyst antibody. Furthermore, culturing and encysting trophozoites in low cholesterol-containing medium interfered with gLR assembly and encystation. Cholesterol supplementation rescued the effect of low cholesterol and recovered the cyst production by $\sim 75 \%$, which reacted mostly with the cyst antibody [64•]. These reports support the idea that the assembly of 
glycosphingolipid (GSL-i.e., GM1) and cholesterolenriched gLRs is important for recruiting signaling proteins and lipids that participate in the encystation processes.

\section{Conclusion}

Encystation, or cyst formation, is an important step in the giardial life cycle. By forming cysts, Giardia can survive outside its hosts for months or years. Many investigators have studied encystation and identified various structural and regulatory proteins, as well as carbohydrates, that are important for this process. Recent reports (discussed in this review) indicate that lipid molecules also play a role during encystation. This is interesting because earlier studies demonstrated that Giardia has a limited lipid-synthesis ability, and the majority of lipids and fatty acids are obtained from the culture medium $[65,66]$. About 30-35 genes related to lipid metabolism are annotated in the Giardia Genome Database (www.GiardiaDB. org), and the majority of these genes are expressed (unpublished). However, only SL-metabolic genes are differentially regulated during encystation $[38 \cdot, 66]$, suggesting that SLs are important for Giardia and play a significant role during cyst formation. Recent findings demonstrate that SLs, especially GSLs (GM1), also contribute in assembling cholesterol-enriched gLRs, and that cholesterol and neuraminidase (sialidase) inhibitors (nystatin, filipin-III, and oseltamivir) disassemble gLRs and inhibit encystation [64•]. A hypothetical model shown in Fig. 2 further elaborates the possible connection between gLRs and SL metabolism in this organism. It is possible that encystation is initiated by gLRs, which send signals downstream of the plasma membranes that upregulate various encystation-specific genes, proteins, and SLs, including gGlcT1. Because ceramide is not synthesized by Giardia, the majority of ceramide is endocytosed by clathrin-coated vesicles [51•] and utilized by gGlcT1 to produce GlcCer and other GSLs to drive the process of encystation and cyst production [57•]. Future work will focus on further analysis of gLR proteins and lipids and how they interact with SL pathways. In addition, both SL pathways and gLRs can be used as targets for development of more potent and effective therapies to control giardiasis. This is important because the anti-giardial drugs that are currently available are not specific against this parasite and may produce side effects.

Acknowledgments The authors' laboratory is supported by a grant (no. R01AI 095667) from the National Institutes of Health (NIH).

\section{Compliance with Ethics Guidelines}

Conflict of Interest Tavis L. Mendez, Atasi De Chatterjee, Trevor Duarte, Joaquin De Leon, Leobarda Robles-Martinez, and Siddhartha Das declare that they have no conflict of interest.
Human and Animal Rights and Informed Consent This article does not contain any studies with human or animal subjects performed by any of the authors.

\section{References}

Papers of particular interest, published recently, have been highlighted as:

- Of importance

1. Adam RD. Biology of Giardia lamblia. Clin Microbiol Rev. 2001;14(3):447-75.

2. Painter JE, Gargano JW, Collier SA, Yoder JS. Giardiasis surveillance-United States, 2011-2012. MMWR Surveill Summ. 2015;64 Suppl 3:15-25.

3. Yoder JS, Gargano JW, Wallace RM, Beach MJ. Centers for Disease Control and Prevention. Giardiasis surveillance-United States, 2009-2010. MMWR Surveill Summ. 2012;61(5):13-23.

4. Yoder JS, Harral C, Beach MJ. Centers for Disease Control and Prevention. Giardiasis surveillance-United States, 2006-2008. MMWR Surveill Summ. 2010;59(6):15-25.

5. Ekdahl K, Andersson Y. Imported giardiasis: impact of international travel, immigration, and adoption. Am J Trop Med Hyg. 2005;72(6):825-30.

6. Dorny P, Praet N, Deckers N, Gabriel S. Emerging food-borne parasites. Vet Parasitol. 2009;163(3):196-206.

7. Bingham AK, Meyer EA. Giardia excystation can be induced in vitro in acidic solutions. Nature. 1979;277(5694):301-2.

8. Boucher SE, Gillin FD. Excystation of in vitro-derived Giardia lamblia cysts. Infect Immun. 1990;58(11):3516-22.

9. Gillin FD, Reiner DS, Gault MJ, Douglas H, Das S, Wunderlich A, et al. Encystation and expression of cyst antigens by Giardia lamblia in vitro. Science. 1987;235(4792):1040-3.

10. Kane AV, Ward HD, Keusch GT, Pereira ME. In vitro encystation of Giardia lamblia: large-scale production of in vitro cysts and strain and clone differences in encystation efficiency. J Parasitol. 1991;77(6):974-81.

11. Dawson SC, House SA. Imaging and analysis of the microtubule cytoskeleton in Giardia. Methods Cell Biol. 2010;97:307-39.

12. Ghosh S, Frisardi M, Rogers R, Samuelson J. How Giardia swim and divide. Infect Immun. 2001;69(12):7866-72.

13. Halliez MC, Buret AG. Extra-intestinal and long term consequences of Giardia duodenalis infections. World J Gastroenterol. 2013;19(47):8974-85.

14. Holberton DV. Mechanism of attachment of Giardia to the wall of the small intestine. Trans R Soc Trop Med Hyg. 1973;67(1):29-30.

15. Benchimol MDS, De Souza W. The ultrastructure of giardia during growth and differentiation. In: Hugo D, Luján SS, editors. Giardia: a model organism. Vienna: Springer; 2011.

16. Sener K, Shen Z, Newburg DS, Jarroll EL. Amino sugar phosphate levels in Giardia change during cyst wall formation. Microbiology. 2004;150(Pt 5):1225-30.

17. Ratner DM, Cui J, Steffen M, Moore LL, Robbins PW, Samuelson J. Changes in the N-glycome, glycoproteins with Asn-linked glycans, of Giardia lamblia with differentiation from trophozoites to cysts. Eukaryot Cell. 2008;7(11):1930-40.

18. Erlandsen SL, Bemrick WJ, Schupp DE, Shields JM, Jarroll EL, Sauch JF, et al. High-resolution immunogold localization of Giardia cyst wall antigens using field emission SEM with secondary and backscatter electron imaging. J Histochem Cytochem. 1990;38(5):625-32. 
19. Chatterjee A, Carpentieri A, Ratner DM, Bullitt E, Costello CE, Robbins PW, et al. Giardia cyst wall protein 1 is a lectin that binds to curled fibrils of the GalNAc homopolymer. PLoS Pathog. 2010;6(8):e1001059.

20. Morf L, Spycher C, Rehrauer H, Fournier CA, Morrison HG, Hehl $\mathrm{AB}$. The transcriptional response to encystation stimuli in Giardia lamblia is restricted to a small set of genes. Eukaryot Cell. 2010;9(10):1566-76.

21. Birkeland SR, Preheim SP, Davids BJ, Cipriano MJ, Palm D, Reiner DS, et al. Transcriptome analyses of the Giardia lamblia life cycle. Mol Biochem Parasitol. 2010;174(1):62-5.

22. Faso C, Bischof S, Hehl AB. The proteome landscape of Giardia lamblia encystation. PLoS One. 2013;8(12):e83207.

23. Lauwaet T, Davids BJ, Torres-Escobar A, Birkeland SR, Cipriano MJ, Preheim SP, et al. Protein phosphatase 2A plays a crucial role in Giardia lamblia differentiation. Mol Biochem Parasitol. 2007;152(1):80-9.

24. Mowatt MR, Lujan HD, Cotten DB, Bowers B, Yee J, Nash TE, et al. Developmentally regulated expression of a Giardia lamblia cyst wall protein gene. Mol Microbiol. 1995;15(5):955-63.

25. Lujan HD, Mowatt MR, Conrad JT, Bowers B, Nash TE. Identification of a novel Giardia lamblia cyst wall protein with leucine-rich repeats: implications for secretory granule formation and protein assembly into the cyst wall. J Biol Chem. 1995;270(49):29307-13.

26. Gottig N, Elias EV, Quiroga R, Nores MJ, Solari AJ, Touz MC, et al. Active and passive mechanisms drive secretory granule biogenesis during differentiation of the intestinal parasite Giardia lamblia. J Biol Chem. 2006;281(26):18156-66.

27. Sun CH, Palm D, McArthur AG, Svard SG, Gillin FD. A novel Myb-related protein involved in transcriptional activation of encystation genes in Giardia lamblia. Mol Microbiol. 2002;46(4): 971-84.

28. Chen YH, Su LH, Huang YC, Wang YT, Kao YY, Sun CH. UPF1, a conserved nonsense-mediated mRNA decay factor, regulates cyst wall protein transcripts in Giardia lamblia. PLoS One. 2008;3(10): e3609.

29. Reiner DS, Hetsko ML, Meszaros JG, Sun CH, Morrison HG, Brunton LL, et al. Calcium signaling in excystation of the early diverging eukaryote, Giardia lamblia. J Biol Chem. 2003;278(4): 2533-40.

30. Faso C, Hehl AB. Membrane trafficking and organelle biogenesis in Giardia lamblia: use it or lose it. Int J Parasitol. 2011;41(5):47180.

31. Reiner DS, McCaffery M, Gillin FD. Sorting of cyst wall proteins to a regulated secretory pathway during differentiation of the primitive eukaryote, Giardia lamblia. Eur J Cell Biol. 1990;53(1):14253.

32. Hehl AB, Marti M. Secretory protein trafficking in Giardia intestinalis. Mol Microbiol. 2004;53(1):19-28.

33. Marti M, Hehl AB. Encystation-specific vesicles in Giardia: a primordial Golgi or just another secretory compartment? Trends Parasitol. 2003;19(10):440-6.

34. de Souza W. Secretory organelles of pathogenic protozoa. An Acad Bras Cienc. 2006;78(2):271-91.

35. McCaffery JM, Faubert GM, Gillin FD. Giardia lamblia: traffic of a trophozoite variant surface protein and a major cyst wall epitope during growth, encystation, and antigenic switching. Exp Parasitol. 1994;79(3):236-49.

36. McCaffery JM, Gillin FD. Giardia lamblia: ultrastructural basis of protein transport during growth and encystation. Exp Parasitol. 1994; 79(3):220-35.

37. Bittencourt-Silvestre J, Lemgruber L, de Souza W. Encystation process of Giardia lamblia: morphological and regulatory aspects. Arch Microbiol. 2010;192(4):259-65.
38. Hernandez Y, Shpak M, Duarte TT, Mendez TL, Maldonado RA, Roychowdhury S, et al. Novel role of sphingolipid synthesis genes in regulating giardial encystation. Infect Immun. 2008;76(7):2939 49. This paper describes the differential expressions of sphingolipid genes during encystation. Also, the inhibitors of serine-palmitoyltransferase and glucosylceramide transferase are shown to inhibit ceamide endocytsosis and cyst production.

39. Sonda S, Stefanic S, Hehl AB. A sphingolipid inhibitor induces a cytokinesis arrest and blocks stage differentiation in Giardia lamblia. Antimicrob Agents Chemother. 2008;52(2):563-9. The authors show here that PPMP, an inhibitor of glucosylceramide synthase, arrests cytokinesis of the trophozoite and its transformation into a cyst.

40. Liu YY, Hill RA, Li YT. Ceramide glycosylation catalyzed by glucosylceramide synthase and cancer drug resistance. Adv Cancer Res. 2013;117:59-89.

41. Liour SS, Yu RK. Differential effects of three inhibitors of glycosphingolipid biosynthesis on neuronal differentiation of embryonal carcinoma stem cells. Neurochem Res. 2002;27(11):1507-12.

42. Lee L, Abe A, Shayman JA. Improved inhibitors of glucosylceramide synthase. J Biol Chem. 1999;274(21):14662-9.

43. Kok JW, Babia T, Filipeanu CM, Nelemans A, Egea G, Hoekstra D. PDMP blocks brefeldin A-induced retrograde membrane transport from golgi to ER: evidence for involvement of calcium homeostasis and dissociation from sphingolipid metabolism. J Cell Biol. 1998;142(1):25-38.

44. Reiner DS, Ankarklev J, Troell K, Palm D, Bernander R, Gillin FD, et al. Synchronisation of Giardia lamblia: identification of cell cycle stage-specific genes and a differentiation restriction point. Int $\mathrm{J}$ Parasitol. 2008;38(8-9):935-44.

45. Farthing MJ, Keusch GT, Carey MC. Effects of bile and bile salts on growth and membrane lipid uptake by Giardia lamblia: possible implications for pathogenesis of intestinal disease. J Clin Invest. 1985;76(5):1727-32.

46. Gillin FD, Gault MJ, Hofmann AF, Gurantz D, Sauch JF. Biliary lipids support serum-free growth of Giardia lamblia. Infect Immun. 1986;53(3):641-5.

47. Mohareb EW, Rogers EJ, Weiner EJ, Bruce JI. Giardia lamblia: phospholipid analysis of human isolates. Ann Trop Med Parasitol. 1991;85(6):591-7.

48. Lanfredi-Rangel A, Kattenbach WM, Diniz Jr JA, de Souza W. Trophozoites of Giardia lamblia may have a Golgi-like structure. FEMS Microbiol Lett. 1999;181(2):245-51.

49. Stevens TL, Gibson GR, Adam R, Maier J, Allison-Ennis M, Das S. Uptake and cellular localization of exogenous lipids by Giardia lamblia, a primitive eukaryote. Exp Parasitol. 1997;86(2):133-43.

50. Stefanic S, Morf L, Kulangara C, Regos A, Sonda S, Schraner E, et al. Neogenesis and maturation of transient Golgi-like cisternae in a simple eukaryote. J Cell Sci. 2009;122(Pt 16):2846-56.

51. Hernandez Y, Castillo C, Roychowdhury S, Hehl A, Aley SB, Das S. Clathrin-dependent pathways and the cytoskeleton network are involved in ceramide endocytosis by a parasitic protozoan, Giardia lamblia. Int J Parasitol. 2007;37(1):21-32. This paper demonstrates that Giardia uses clathrin-dependent endocytic pathways to import exogenous ceramide from the culture medium.

52. Stefanic S, Spycher C, Morf L, Fabrias G, Casas J, Schraner E, et al. Glucosylceramide synthesis inhibition affects cell cycle progression, membrane trafficking, and stage differentiation in Giardia lamblia. J Lipid Res. 2010;51(9):2527-45. This paper describes how glucosylceramide synthesis is important for giardial encystation and blocking of its synthesis by PPMP (an inhibitor of glucosylceramide synthase) affects cell cycle progression, membrane trafficking, and encystation.

53. Das S, Reiner DS, Zenian J, Hogan DL, Koss MA, Wang CS, et al. Killing of Giardia lamblia trophozoites by human intestinal fluid in vitro. J Infect Dis. 1988;157(6):1257-60. 
54. Deguchi M, Gillin FD, Gigli I. Mechanism of killing of Giardia lamblia trophozoites by complement. J Clin Invest. 1987;79(5): 1296-302.

55. Lauwaet T, Davids BJ, Reiner DS, Gillin FD. Encystation of Giardia lamblia: a model for other parasites. Curr Opin Microbiol. 2007;10(6):554-9.

56. Isaac-Renton JL, Philion JJ. Factors associated with acquiring giardiasis in British Columbia residents. Can J Public Health. 1992;83(2):155-8.

57. Mendez TL, De Chatterjee A, Duarte TT, Gazos-Lopes F, RoblesMartinez L, Roy D, et al. Glucosylceramide transferase activity is critical for encystation and viable cyst production by an intestinal protozoan, Giardia lamblia. J Biol Chem. 2013;288(23):16747-60. This paper illustrates that glucosylceramide synthase (also known as glucosylceramide tranferase) regulates ESV biogenesis and viable cyst production by Giardia.

58. Gillin FD, Boucher SE, Rossi SS, Reiner DS. Giardia lamblia: the roles of bile, lactic acid, and $\mathrm{pH}$ in the completion of the life cycle in vitro. Exp Parasitol. 1989;69(2):164-74.

59. Cary LA, Cooper JA. Molecular switches in lipid rafts. Nature. 2000;404(6781):945-947.

60. Simons K, Ehehalt R. Cholesterol, lipid rafts, and disease. J Clin Invest. 2002;110(5):597-603.
61. Simons K, Toomre D. Lipid rafts and signal transduction. Nat Rev Mol Cell Biol. 2000;1(1):31-9.

62. Stuermer CA. Microdomain-forming proteins and the role of the reggies/flotillins during axon regeneration in zebrafish. Biochim Biophys Acta. 2011;1812(3):415-22.

63. Sonnino S, Prinetti A, Mauri L, Chigorno V, Tettamanti G. Dynamic and structural properties of sphingolipids as driving forces for the formation of membrane domains. Chem Rev. 2006;106(6):2111-25.

64. De Chatterjee A, Mendez TL, Roychowdhury S, Das S. The assembly of GM1 glycolipid- and cholesterol-enriched raft-like membrane microdomains is important for giardial encystation. Infect Immun. 2015;83(5):2030-42. This paper demonstrates that Giardia has the ability to assemble cholesterol and glycolipidenriched lipid rafts and that disassembly of lipid rafts inhibits encystation.

65. Jarroll EL, Bingham AK, Meyer EA. Effect of chlorine on Giardia lamblia cyst viability. Appl Environ Microbiol. 1981;41(2):483-7.

66. Yichoy M, Duarte TT, De Chatterjee A, Mendez TL, Aguilera KY, Roy D, et al. Lipid metabolism in Giardia: a post-genomic perspective. Parasitology. 2011;138(3): $267-78$. 